\title{
EXTREME ULTRAVIOLET OBSERVATIONS OF ACTIVE REGIONS IN THE SOLAR CORONA
}

\author{
W. M. BURTON \\ (Culham Laboratory, Abingdon, Berks., England)
}

\begin{abstract}
A BSTRACT
The coronal features associated with solar active regions can be observed by recording images of the Sun at extreme ultraviolet (XUV) wavelengths. Pinhole cameras have been flown on stabilized sun-pointing 'Skylark' rockets to obtain broad-waveband XUV solar images. These images show localised emission from high-temperature regions located in the corona above calcium-plage areas. An improved design of pinhole camera, which uses a plane-diffraction grating to give increased spectral resolution, has recorded spectroheliograms in several intense solar lines including $\mathrm{HeII}$ $(304 \AA)$, FeIX-XI (180 $\AA$ ), and Six-XII (50 $\AA$ ). Estimates are made of the size and brightness of the coronal emission region associated with a developing calcium-plage area.
\end{abstract}

\section{Introduction}

The structure of a solar active region can be studied by recording spectroheliograms in various spectral lines which are formed at different temperatures in the Sun. Each line then defines a different level in the solar atmosphere, so that a detailed picture can be obtained showing the three-dimensional structure of the active region.

Spectroheliograms recorded in the hydrogen $\mathrm{H} \alpha$ and $\mathrm{Ca} \mathrm{K}$ lines are valuable for studying the chromospheric structure of active regions, but they provide no information about the high-temperature coronal features which may be associated with the calcium plages.

In order to study the coronal regions which extend above a solar centre of activity (CA), the observations should be made using a wavelength at which the brightness of the active region is greater than that of the undisturbed solar disk. This criterion is satisfied in two distinct wavebands, firstly at $10-\mathrm{cm}$ radio wavelengths and secondly at much shorter wavelengths in the X-ray and extreme ultraviolet (XUV) region of the spectrum.

This paper describes some observations of the coronal features associated with active regions, obtained by recording solar images in the $20-400 \AA$ XUV wavelength region.

\section{Broad-band XUV Solar Images}

A simple pinhole camera can be used for recording photographic images of the Sun at XUV wavelengths (Blake et al., 1963). The camera used for the present observations

Kiepenheuer (ed.), Structure and Development of Solar Active Regions, 395-402. C. I.A.U. 
had an optical length of $25 \mathrm{~cm}$ and so formed a solar image $2.3 \mathrm{~mm}$ in diameter. The angular resolution of the camera was about $2 \mathrm{~min}$ of arc as determined by the pinhole aperture of $0.15 \mathrm{~mm}$. Special XUV sensitive photographic film (Kodak-Pathé SC 5 and SC7) was used to record the solar images.

The cameras have been carried on stabilized 'Skylark' rockets launched from Woomera, South Australia. A peak height of about $200 \mathrm{~km}$ can be reached, which provides an exposure period of $200 \mathrm{sec}$ during the part of the flight when atmospheric absorption is reduced sufficiently for XUV studies of the Sun. An attitude-control system stabilized the rocket payload relative to the Sun, giving spatial resolution of about 1 min of arc on the solar disk, together with a few degrees of disk rotation during the exposure period (Cope, 1964).

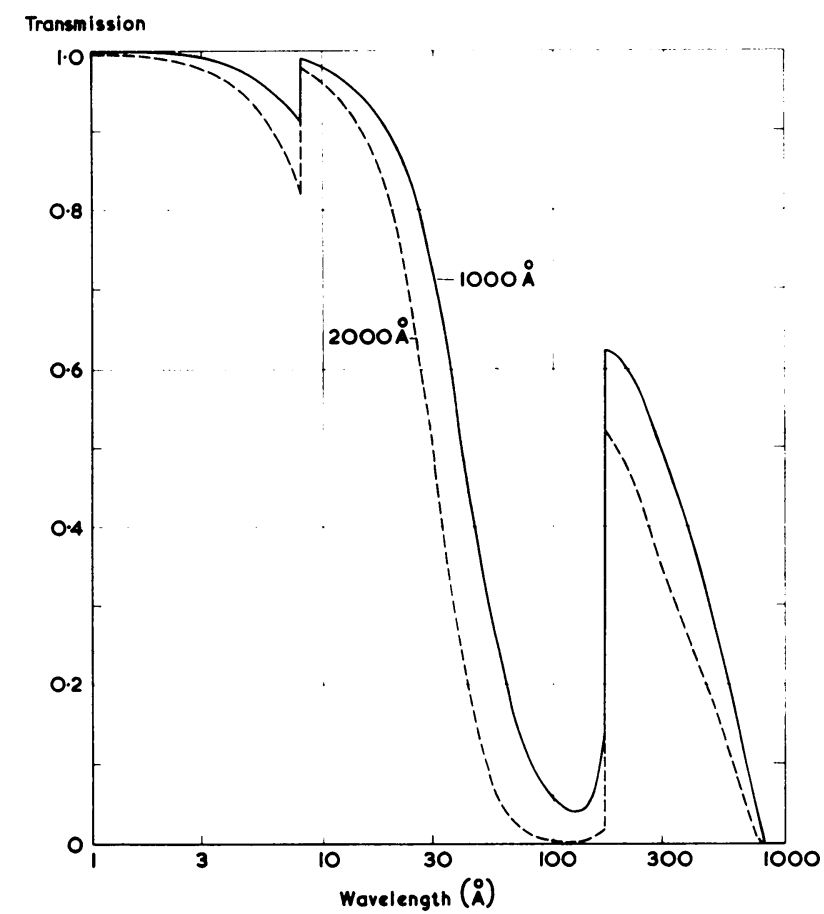

FIG. 1. Calculated transmission curves for aluminium filters of $1000 A$ and $2000 A$ thickness.

The spectral response of the camera is set by a thin film of aluminium which is placed over the pinhole aperture to exclude visible light while transmitting XUV radiation. Figure 1 shows the variation of transmission with wavelength for typical aluminium filters. A $2000-\AA$ thickness filter is opaque at wavelengths longer than $800 \AA$, but has two useful transmission bands, one from $800 \AA$ to the L-edge at $170 \AA$ and the other extending from $80 \AA$ down to the $\mathrm{X}$-ray region. In practice, the observed 
wavebands are limited further by residual atmospheric absorption and by the form of the solar emission spectrum. The effective wavebands producing the solar image for this type of camera are consequently $20-70 \AA$ and $170-400 \AA$.

A camera with this broad-band response was flown on December 17, 1964 and again on April 9, 1965. Solar images were formed by a blend of XUV emission lines, of which the most important were spectral lines of SiX-XII (40-60 $\AA$ ) and FeIX-XII (170-220 $\AA$ ). These ions have their maximum equilibrium population in a thermal plasma at a temperature of about $2 \times 10^{6}{ }^{\circ} \mathrm{K}$ and the emission therefore defines regions of enhanced density at this typical coronal temperature.

The XUV image recorded on December 17, 1964 is shown in Figure 2 together with the Fraunhofer Institute solar map for the same day. The image is displayed
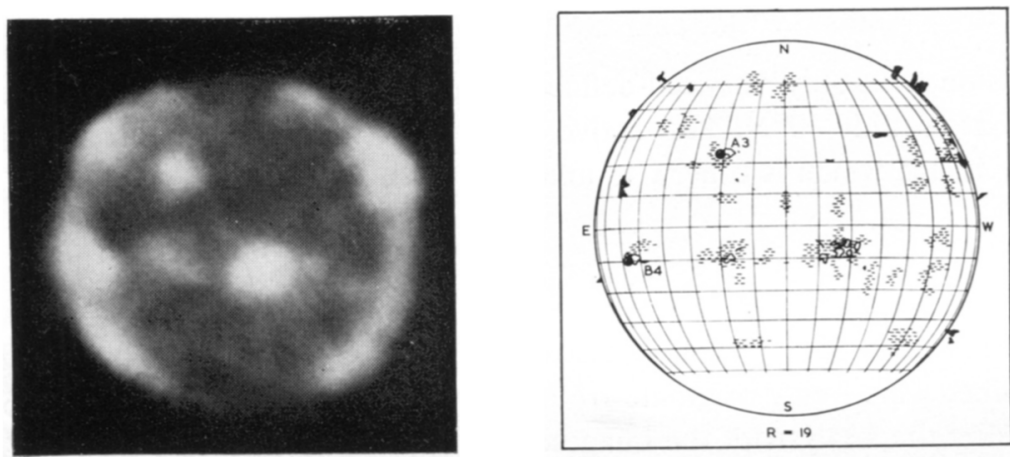

FIG. 2. Solar XUV image recorded on December 17, 1964, together with Fraunhofer Institute solar map for the same day.

with North at the top and the East limb to the left. Although near the minimum in the solar cycle, the Sun was relatively active at the time of observation. Strong limb brightening is present at middle latitudes but the polar regions are very dark. Several distinct active regions can be seen in the XUV image, with some localised darker areas between them. All of the XUV emission regions correspond with calcium-plage areas. The age range of these plages is very large, suggesting that the coronal emission region is a persistent feature with a lifetime similar to that of the calcium plage.

The XUV emission region on the NE limb is associated with a calcium-plage area which was not visible on the solar disk until the day following this observation. At the time of the rocket flight the plage area was located about $12^{\circ}$ behind the NE solar limb. This implies that the coronal emission source was located at least $10000 \mathrm{~km}$ above the solar photosphere. Similarly, the XUV source on the NW limb persists strongly although part of the underlying calcium plage has moved behind the limb.

On April 9,1965 a similar camera was used to record the XUV image which is shown in Figure 3 with the solar map for that day. The Sun was rather inactive on 

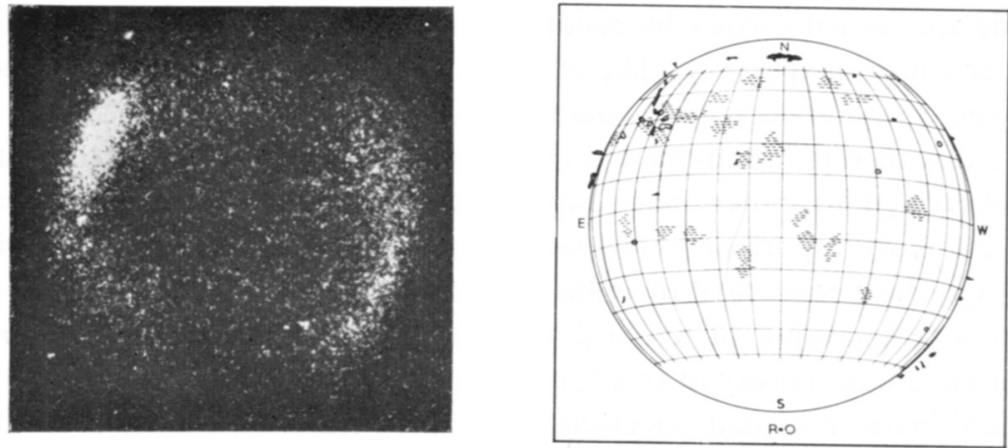

FIG. 3. Solar XUV image recorded on April 9, 1965, together with Fraunhofer Institute solar map for the same day.

this occasion with only one well-defined plage area, which was located close to the $\mathrm{NE}$ limb at latitude $30^{\circ} \mathrm{N}$. This active region coincides with an emission feature in the XUV image which is otherwise underexposed.

\section{XUV Spectroheliograms}

The broad-band XUV images described above are formed by a blend of several spectral lines which may originate from different levels in the solar atmosphere, so complicating the analysis of the images. In an attempt to increase the spectral resolution, an improved pinhole camera has been developed which uses a plane diffraction grating at grazing incidence to provide wavelength dispersion (Burton, 1965). An outline diagram of this grating-pinhole camera is shown in Figure 4. The camera

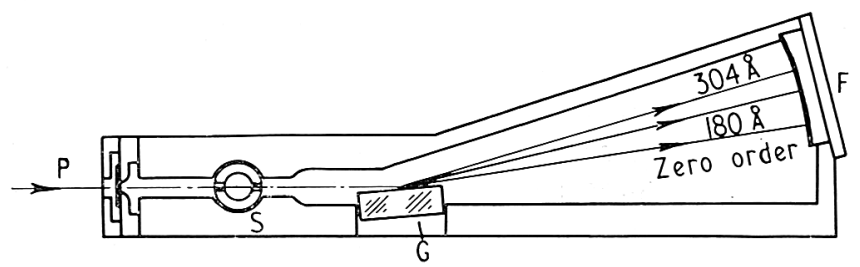

FIG. 4. Grating-pinhole camera forming dispersed XUV solar images. $P=$ pinhole aperture covered by aluminium filter. $S=$ shutter to control exposure duration. $G=600$ line/mm plane diffraction grating. $F=$ photographic film to record images.

length is again $25 \mathrm{~cm}$, so that the solar image diameter is about $2.3 \mathrm{~mm}$. Since the reciprocal dispersion is only $20 \AA / \mathrm{mm}$, the camera forms overlapping images in spectral lines, which are spaced by less than $50 \AA$. The grating is blazed for optimum efficiency at $180 \AA$, the wavelength of a group of intense spectral lines of highly ionized iron which are prominent in the solar XUV spectrum. 
The grating-pinhole camera forms a broad-band XUV image at the zero-order position and also a first-order spectrum between $40 \AA$ and $400 \AA$ in which solar images are formed by each strong emission line. Figure 5 shows composite spectroheliograms recorded by this instrument on two flights: April 9, and October 20, 1965. Wavelength dispersion is in the direction of the solar North-South polar axis and the West limb is at the top of each dispersed spectrum. Spectral resolution is limited by overlapping images but several separate features can be seen. The He II resonance line at $304 \AA$ which is formed mainly in the chromosphere, shows a uniform disk image

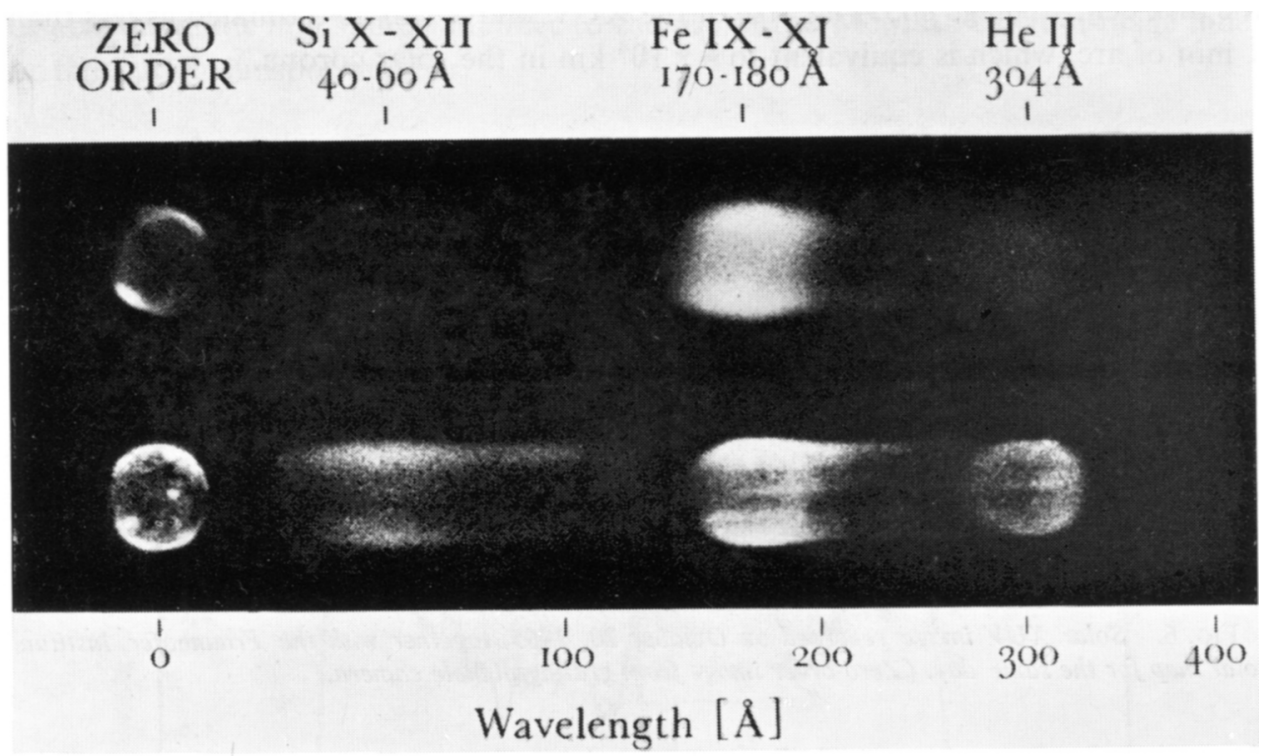

FIG. 5. Composite spectroheliograms (40-400 A) from grating-pinhole camera. (a) April 9, 1965. (b) October 20, 1965.

with slight limb brightening at this resolution. The most intense of the dispersed images is that formed by the group of intense ionized iron lines FeIX-XI at $180 \AA$. Between $40 \AA$ and $60 \AA$ emission is seen from overlapping images in spectral lines of Si X-XII.

Several active regions can be seen in the October 20,1965 images, all of which coincide with calcium-plage areas. Between the bright regions, the background-disk intensity is not uniform but shows localised dark regions which are comparable in area to the active regions. The bright source close to the disk centre (McMath plage no. 8032) is a developing CA, which was the site of a small solar flare recorded on the day before these XUV observations were made. In the HeII $304 \AA$ image, this is the only active region which can be detected with certainty, but in the other lines, formed at higher levels, this CA is similar in intensity to the other active regions. 
The zero-order image is formed primarily by the group of ionized iron lines at $180 \AA$ and is shown in more detail in Figure 6. Equatorial limb brightening is very strong since the emission comes mainly from the corona which is optically thin in these wavelengths. Emission can be seen from most of the solar disk but is particularly prominent across the middle latitude region in the Northern hemisphere. Several localised emission regions can be seen in this image and their size can be estimated by correcting the observed size for effects of camera resolution and pointing errors during the exposure period. The measurement is difficult for active regions close to the limb where the effects of limb brightening are important, but for the CA close to the disk centre (plage 8032) the size of the XUV source can be estimated as less than $1 \mathrm{~min}$ of arc, which is equivalent to $4 \times 10^{4} \mathrm{~km}$ in the solar corona.
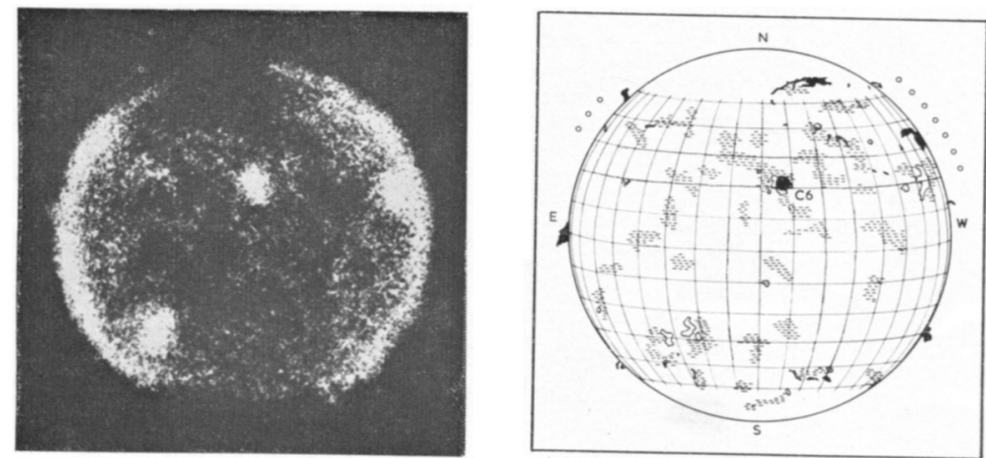

FIG. 6. Solar XUV image recorded on October 20,1965, together with the Fraunhofer Institute solar map for the same day. (Zero-order image from grating-pinhole camera.)

By densitometer measurements of the photographic image, the XUV brightness of this active region is estimated to be about five times greater than the mean quiet solar disk background. This figure is an order of magnitude lower than the values obtained in similar observations using X-rays in the wavelength region below $60 \AA$ (Blake et al., 1963; Pounds and Russell, 1966). The observed brightness is related to the coronal electron density $\left(N_{\mathrm{e}}\right)$, and for each spectral line is proportional to $\left(N_{\mathrm{e}}\right)^{2} h$, where $h$ is a characteristic height for the emission region. The quantity $\left(N_{\mathrm{e}}\right)^{2} h$ is apparently greater in the active region than in the quiet undisturbed corona by a factor 5 in the XUV lines characteristic of a temperature of about $2 \times 10^{6}{ }^{\circ} \mathrm{K}$, and by a factor 50 in $\mathrm{X}$-ray emission, which is formed at a rather higher temperature.

This result could be interpreted in terms of the density variation within the coronal active region if a reliable model of the temperature structure of a CA were available, but at the present time there is insufficient information to interpret the intensity data in this way. 


\section{Standardised Measurements on XUV Images}

Solar images at XUV and X-ray wavelengths are now obtained regularly by several laboratories. The analysis of these images is not simple, and the data are seldom made available in a convenient form for use by those interested in solar physics. A standard procedure for measurement and definition of the XUV brightness of solar active regions has recently been proposed (Allen, 1967). The measurement of CA flux for particular XUV or X-ray wavebands is made in terms of 'solar disk units' to provide dimensionless numbers which can be used to describe the XUV importance of active regions in a similar manner to the use of the McMath calcium-plage index or the Zurich sunspot number.

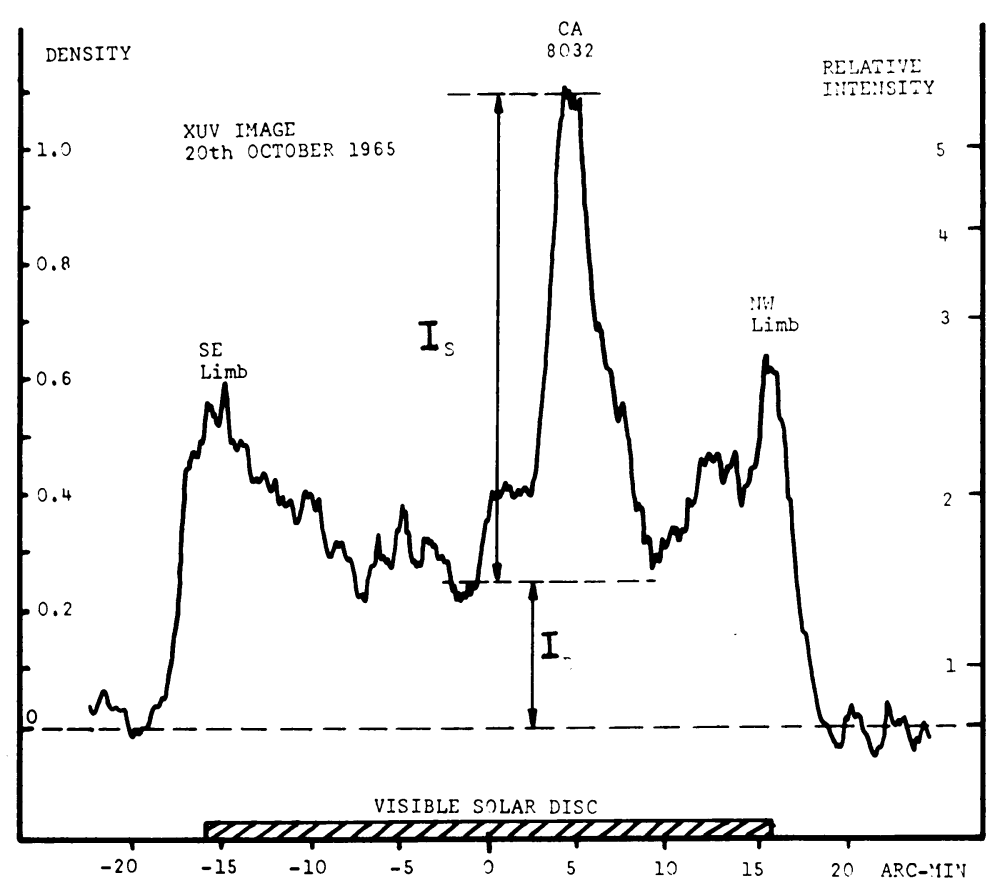

FIG. 7. Densitometer scan of XUV solar image, showing method of measurement and definition of CA flux in solar disk units.

The measurement procedure is shown in Figure 7 with reference to a densitometer scan across the solar image for October 20, 1967 shown in Figure 6. Calibration of the photographic film is required to determine a characteristic curve which is used to convert the density measurements to relative intensities. The flux from each $\mathrm{CA}$ source then is estimated from the expression $F_{\mathrm{s}}=\int I_{\mathrm{s}} \mathrm{d} A$, where $I_{\mathrm{s}}$ represents the intensity of the source in excess of the quiet disk background intensity. The quantity 
$F_{\mathrm{s}}$ is standardised by measuring $I_{\mathrm{s}}$ in units of $I_{D}$, the intensity at the centre of the quiet solar disk, and by measuring the apparent source area $\mathrm{d} A$ in units of the projected solar disk radius $R_{\odot}$. Units based on $I_{D}$ and $R_{\odot}$ are then called 'disk units'.

For the images described in this paper the quantity $F_{\mathrm{s}}$ for the XUV emission from active regions individual CA sources varies between 0.01 and $1 \cdot 0$. For $\mathrm{X}$-ray measurements the value of $F_{\mathrm{s}}$ in disk units is greater due to the increased contrast between the CA intensity and quiet-disk intensity. At shorter X-ray wavelengths the value of $I_{D}$ may be too low for reliable measurements, in which case the total solar flux would provide a better reference intensity for the standardisation of $F_{\mathrm{s}}$ measurements.

\section{References}

Allen, C.W. (1967) Private communication.

Blake, R. L., Chubb, T. A., Friedman, H., Unzicker, A.E. (1963) Astrophys. J., 137, 3.

Burton, W.M. (1965) J.Sci.Instrum., 42, 477.

Cope, P.E.G. (1964) J. Brit. Interplan. Soc., 19, 285.

Pounds, K.A., Russell, P.C. (1966) in Space Research, VI, Spartan Books, New York, p. 34. 